\title{
Functional analysis and clinical significance of the isocitrate dehydrogenase 2 gene in papillary thyroid carcinoma
}

\author{
Jun Zhang ${ }^{1-4, *}$ \\ Linfei $\mathrm{Hu}^{2-5}$,* \\ Huijuan Wang ${ }^{2-5}$ \\ Jingtai $\mathrm{Zhi}^{2-5}$ \\ Xiukun $\mathrm{Hou}^{2-5}$ \\ $\mathrm{Yu} \mathrm{Wu}{ }^{6}$ \\ Xiangqian Zheng ${ }^{2-5}$ \\ Ming $\mathrm{Gao}^{2-5}$
}

'Department of Breast Surgery, Tianjin Medical University Cancer Institute and Hospital, Tianjin Medical University,

Tianjin, People's Republic of China;

${ }^{2}$ Department of National Clinical

Research Center for Cancer, Tianjin

Medical University Cancer Institute and

Hospital, Tianjin Medical University,

Tianjin, People's Republic of China;

${ }^{3}$ Department of Tianjin's Clinical

Research Center for Cancer, Tianjin

Medical University Cancer Institute and

Hospital, Tianjin Medical University,

Tianjin, People's Republic of China;

${ }^{4}$ Department of Key Laboratory of

Cancer Prevention and Therapy, Tianjin

Medical University Cancer Institute and

Hospital, Tianjin Medical University,

Tianjin, People's Republic of China;

${ }^{5}$ Department of Thyroid and Neck

Tumor, Tianjin Medical University

Cancer Institute and Hospital, Tianjin

Medical University, Tianjin, People's

Republic of China; ${ }^{6}$ Department of

Head and Neck Surgery, Fujian Cancer

Hospital, Fujian Medical University

Cancer Hospital, Fuzhou, People's

Republic of China

*These authors contributed equally to this work

Correspondence: Ming Gao; Xiangqian Zheng

Department of Thyroid and Neck Tumor,

National Clinical Research Center for

Cancer, Tianjin Medical University Cancer

Institute and Hospital, Tianjin, 300060,

People's Republic of China

Email headandneck2008@126.com
This article was published in the following Dove Press journal:

Cancer Management and Research

Objective: Mitochondrial NADP+-dependent isocitrate dehydrogenase 2 (IDH2) is a major producer of mitochondrial NADPH. IDH2-related research has focused on its mutation mechanism and its clinical significance, but the role of wild-type IDH2 in carcinoma remains controversial. Altered IDH2 levels have been identified in several types of carcinomas. However, the significance and expression of IDH2 in thyroid cancer remains unknown.

Methods: We examined the expression of IDH2 in thyroid cancer and adjacent normal tissues using quantitative real-time PCR (qRT-PCR), immunohistochemical (IHC) staining analyses, and western blot analysis with frozen tissues. The relationship between IDH2 and the clinicopathological features of thyroid cancer was analyzed by IHC. Subsequently, we investigated the function of wild-type IDH2 in thyroid cancer cells in vitro.

Results: We found that the mRNA expression and protein levels of IDH2 were higher in tumor than in adjacent tissues, when evaluated by qRT-PCR, western blot, and IHC analyses. Tumor size, T stage, lymph node metastasis, and TNM stage showed significant differences between the IDH2 high expression and low expression groups. Multiple logistic regression analyses indicated that tumor size and IDH2 expression were significantly correlated with the occurrence of neck LNM. Furthermore, CCK8 levels, colony formation, and invasive cell number were decreased in the sh-IDH2 groups. The upregulation of IDH2 in thyroid cancer cells showed opposite effects.

Conclusion: Our results indicated that IDH2 may play an important role in the development of thyroid cancer. IDH2 can be used as a potential biomarker for diagnosis and prognosis and may be a potential therapeutic target for thyroid cancer.

Keywords: thyroid papillary carcinoma, IDH2, function analysis, therapeutic target

\section{Introduction}

Carcinoma of the thyroid gland is a common endocrine malignancy. The incidence of thyroid cancer has increased worldwide over the past few decades, especially among women. ${ }^{1-3}$ By 2030, if these recent trends are maintained, thyroid cancer will be the fourth most common cancer. ${ }^{4}$ Papillary thyroid carcinoma (PTC) is the most common histological type of thyroid cancer, accounting for over $90 \%$ of all thyroid malignancies. ${ }^{5}$ Most PTC patients have good prognoses, with a 10 -year survival rate exceeding $95 \% .^{5-7}$ Nevertheless, the incidence of lymph node metastasis can be as high as $20-50 \%,{ }^{8}$ and PTC patients undergoing total thyroidectomy have a regional recurrence rate of $5-20 \%{ }^{9}$ Without therapeutic options, these patients have poor prognoses. ${ }^{10-12}$ Therefore, investigating the molecular 
mechanisms and genetic alterations of PTC are imperative and necessary for thyroid carcinoma.

Metabolic changes are thought to play an important role in tumor development. Otto Warburg found that cancer cells prefer to produce ATP (adenosine triphosphate) by glycolysis rather than by oxidative phosphorylation (OXPHOS) even in the presence of ample oxygen. ${ }^{13,14}$ This finding was later termed the Warburg effect. The recent discovery of mutations targeting metabolic genes in cancer has generated a renewed interest in cancer metabolism. Isocitrate dehydrogenase (IDH) is a key enzyme in the tricarboxylic acid (TCA) cycle. IDHs include 3 members: IDH1, IDH2, and IDH3. IDH1 is located in the cytosol and the peroxisomes, while IDH2 and IDH3 are located in the mitochondria. ${ }^{15,16}$ IDH2 mutations confer a gain-of-function, and lead to neomorphic enzymatic activity. ${ }^{17}$ Wild-type IDH2 catalyzes isocitrate to produce alpha-ketogluatarate $(\alpha-\mathrm{KG})$ and $\mathrm{NADPH} / \mathrm{CO}_{2}$, and the mutant IDH2 catalyzes alpha-ketogluatarate to produce beta-hydroxyglutarate (2-HG). ${ }^{18,19}$ Supra-normal levels of intracellular 2-HG lead to hypermethylation of target genes, which in turn blocks cellular differentiation. ${ }^{20,21}$

In 2009, a mutation in the IDH2 gene at position R172 was first discovered in patients with gliomas, including astrocytomas and oligodendrogliomas. ${ }^{22}$ Subsequently, R140 mutations were found in acute myeloid leukemia (AML). ${ }^{17,19}$ To date, IDH2 mutants have been detected in several malignancies. ${ }^{23,24}$ However, research on IDH2 has primarily focused on its mutation mechanism and clinical significance, and there are very few studies on the function of wild-type IDH2. In addition, the IDH2 gene was examined for mutations in thyroid cancer, but no mutations were found in thyroid cancer. ${ }^{25}$ The alteration of wild-type IDH2 levels has been identified in several types of carcinomas. Wild-type IDH2 has been shown to be overexpressed in testicular and prostate cancers, ${ }^{26}$ endometrial cancer, ${ }^{27}$ and Kashin-Beck disease. ${ }^{28}$ In contrast, wild-type IDH2 is downregulated in gastric cancer (GC) and hepatocellular carcinoma (HCC), and patients with low IDH2 demonstrated a lower 5-year survival rate. ${ }^{29,30}$ However, the significance of IDH2 expression in thyroid cancer is still unknown.

In this study, we examined the expression of key metabolic-related enzymes in thyroid cancer and adjacent normal tissues by quantitative real-time PCR (qRTPCR). We first investigated the expression of IDH2 in thyroid cancer tissues and compared it with its expression in adjacent normal tissues by qRT-PCR and western blot analysis, using frozen tissues. The relationship between IDH2 and the clinicopathological features of thyroid cancer was analyzed by immunohistochemistry (IHC). Subsequently, we investigated the function of wild-type IDH2 in thyroid cancer cells by inhibiting or overexpressing IDH2 via plasmid transfection in in vitro experiments. Our results indicated that IDH2 may play an important role in the development of thyroid cancer. IDH2 can be used as a potential biomarker for diagnosis and prognosis and may be a potential therapeutic target for thyroid cancer.

\section{Materials and methods}

\section{Ethics statement}

All tissue samples were taken with informed consent of the patient. According to the latest revision of the Helsinki declaration, the program of sample analysis was approved by Institutional Review Board of the Tianjin Medical University Cancer Institute and Hospital. The research was approved by Institutional Review Board of the Tianjin Medical University Cancer Institute and Hospital.

\section{Patient and frozen tissue selection}

187 patients with papillary thyroid carcinoma were identified between January 2014 and January 2017 at Tianjin Medical University Cancer Hospital, Tianjin, China. The patient clinicopathological characteristics were obtained retrospectively from the medical records and evaluated as prognostic factors. All patients received thyroidectomy and lymph node area cleaning. The protocol was approved by the Tianjin Medical University Cancer Institute and the Hospital ethics board. Informed consent in writing was obtained from all enrolled patients. Meanwhile, we randomly selected frozen normal and tumor tissues from 21 clinical patients harboring PTC for subsequent RNA and protein extraction.

\section{PCR amplification and sequencing}

We selected the frozen tissue of the 21 patients mentioned above for mutation sequencing. Total RNA was extracted from fresh tissues of papillary thyroid carcinoma and adjacent normal thyroid using TRIzol reagent (Life Technologies) according to the manufacturer's instructions. $500 \mathrm{ng}$ RNA was used for reverse transcription with the PrimeScript RT reagent Kit (Takara). PCR amplification of exon 4 of IDH2 with cDNA, the hotspot of IDH2 mutation in other cancers, was performed using the forward primer IDH2 5'- TGCACTCTAGACT CTACTGCC $-3^{\prime}$ and reverse primer IDH2 5'- ACAAAGT 
CTGTGGCCTTGTAC $-3^{\prime}$ with the annealing temperature at $60{ }^{\circ} \mathrm{C}$ and 35 cycles. The amplified products were sent to the external vendor for Sanger sequencing and alignment with sequences in the gene bank, in which the accession number of IDH2 is NM_002168.2.

\section{Cell lines and cultured}

K-1 and BCPAP human papillary thyroid carcinoma cell lines (both harboring a $\mathrm{BRAF}^{\mathrm{V} 600 \mathrm{E}}$ mutation),KTC-1, TPC-1 and IHH4 human papillary thyroid carcinoma cell lines, $\mathrm{N}$ thy-ori 3-1 human normal thyroid cell line, Cal-62 and $8505 \mathrm{C}$ human thyroid anaplastic carcinoma were purchased from ATCC and appraised. The cells were approved by the Institutional Review Board of the Tianjin Medical University Cancer Institute and Hospital. K-1 was cultured in DMEM (Gibco c11995500bt) supplemented with 10\% FBS (Eallbiou16001dc), 1\% NonEssential Amino Acids Solution (Gibco,11140050), 1\% L-Glutamine (Gibco,25030081) and 1\% PenicillinStreptomycin (Gibco, 10378016, $100 \mathrm{U} / \mathrm{mL}$ penicillin, $100 \mu \mathrm{g} / \mathrm{mL}$ streptomycin). BCPAP was cultured in RPMI 1640 Medium (Gibco c11875500bt) supplemented identically to $\mathrm{K}-1$. The culture conditions of $\mathrm{N}$ thy-ori 3-1, Cal62 , KTC-1 and IHH4 were the same as those for BCPAP, and the culture conditions of $8505 \mathrm{C}$ and TPC-1 were the same as those for $\mathrm{K}-1$ and all cells mentioned above were purchased from ATCC.The cells were maintained in an incubator set to $37{ }^{\circ} \mathrm{C}$ with $5 \% \mathrm{CO} 2$.

\section{Antibodies}

Anti-GAPDH antibody was purchased from Genetex and anti-IDH2 was purchased from Abcam.

\section{Construction of stable cell lines}

Lenti-virus was purchased from Shanghai Genechem Co., LTD. The shRNA-1 sequence was 5'-GATCCCCGTGATG AGATGACCCGTATTATTCAAGAGATAAT ACGGGTC ATCTCATCACTTTTTA-3', and the shRNA-2 sequence was 5'-GATCCCCGAGCACCAAGAACACCATACTTTC AAGAGAAGTATGGTGTTCTTGGTGCTCTTTTTA-3'. Cells were infected with $10 \mathrm{MOI}$ of lenti-virus for $12 \mathrm{~h}$ and after 2 days were selected for in $1 \mu \mathrm{g} / \mathrm{mL}$ puromycin (Abcam) for 14 days After two-weeks in selection medium, cells were grown in standard RPMI 1640 or DMEM media respectively.

\section{Immunohistochemistry}

Formalin-fixed tissue samples were embedded in paraffin and $5 \mu \mathrm{m}$ sections were cut. For immunohistochemistry staining, in brief, the tissue sections on coated slides were dewaxed and subjugated to antigen retrieval by boiling in $10 \mathrm{mM}$ sodium citrate $(\mathrm{pH} 6.0)$ at $130{ }^{\circ} \mathrm{C}$ for $3 \mathrm{~min}$, then pretreated with a $3 \%$ solution of hydrogen peroxide for $30 \mathrm{~min}$, rinsed, and incubated with 5\% normal goat serum for $20 \mathrm{~min}$ as a blocking agent. The sections were incubated with mouse anti-IDH2 antibody (1:100; Abcam) at $4{ }^{\circ} \mathrm{C}$ overnight. The next day, slides were washed in PBS and incubated with the secondary antibody for $30 \mathrm{~min}$ at room temperature. All steps were preceded by rinsing of sections with PBS (pH 7.6). The chromogen was 3,3-diaminobenzidine (DAB). The immunoreactivity of IDH2 in the nucleus of tumor tissues was scored by the H-score method.

\section{Quantitative real-time PCR}

The extraction and reverse transcription of total RNA from cells were as described of that in fresh tissues mentioned above. The cDNA was then used as a template for exponential amplification using SYBR Green/ROX qPCR Master Mix (Thermo scientific) as follows: $95{ }^{\circ} \mathrm{C}$ for $10 \mathrm{~min}$, then 40 cycles of $95^{\circ} \mathrm{C}$ followed by $62^{\circ} \mathrm{C}$ for $1 \mathrm{~min}$. Three replicates were tested in each group and the experiment was performed in triplicate. The sequences of primer pairs were as follows:

human HK1 primer: sense, 5'-AATGCTGGGAAACAA AGGT-3' and antisense, 5'-AGAGGAATCCCTTCTTG GG-3';

human HK2 primer: sense, 5'-GATTGTCCGTAAC ATTCTCATCGA-3' and antisense, 5'-TGTCTTGAGCCG CTCTGAGAT-3';

human IDH1 primer: sense, 5'-CAAGTGACGGAACC CAAAAG-3' and antisense, 5'-ACCCTTAGACAGAG CCATTTG-3';

human IDH2 primer: sense, 5'-TTGGCTGCTTGCAT TAAAGGTT-3' and antisense, 5'-GTTTGGCCTGAGCTA GTTTGA-3';

human PKM2 primer: sense, 5'-CCATTACCAGCG ACCCCACAG-3' and antisense, 5'-GGGCACGTGGGC GGTATCT-3'.

human $\beta$-actin primer: sense, 5'-GATCATTGCTCC TCCTGAGC-3' and antisense, 5'-ACTCCTGCTTGCTG ATCCAC-3'.

\section{Western blotting analysis}

Cells and tissues were lysed in RIPA solution with protease inhibitor PMSF (1mM) for $30 \mathrm{~min}$ on ice. Cell or tissue lysates were centrifuged at $12,000 \mathrm{~g}$ for $15 \mathrm{~min}$ at $4{ }^{\circ} \mathrm{C}$, and the supernatants were collected. Protein concentrations were quantified using the BCA Protein Assay according to the 
manufacturer's instructions. Equal amounts $(20 \mu \mathrm{g})$ of total protein were separated by SDS-PAGE gel (10-12\%) at 70V for $0.5 \mathrm{~h}, 120 \mathrm{~V}$ for $1 \mathrm{~h}$ and transferred to a $0.45 \mu \mathrm{m}$ PVDF membrane at $300 \mathrm{~mA}$ for $60-150 \mathrm{~min}$. After blocking with $5 \%$ non-fat milk in TBST buffer for $1 \mathrm{~h}$ at room temperature, the membranes were incubated with primary antibody at $4{ }^{\circ} \mathrm{C}$ overnight. The membranes were washed three times with TBST buffer and then incubated with peroxidase (HRP)conjugated secondary antibody for $1 \mathrm{~h}$ at room temperature. Specific antibody binding was detected by the Chemiluminescence Kit (Millipore, Plano, TX, USA). Fluorescent signals were detected by a luminescent image analyzer (C-Digit, Gene Company Limited, China).

\section{Cell viability and colony formation assay}

Cell Counting Kit-8 (CCK-8) assay (Dojindo, Kumamoto, Japan) was employed according to the manufacturer's instructions to measure the effects of drugs on the proliferation of papillary thyroid carcinoma cells. Briefly, K-1 and BCPAP cells were seeded into 96-well cell plates (Corning Inc., Corning, USA) with a density of 1000 cells/well in $200 \mu \mathrm{l}$ culture medium, and incubated for $0 \mathrm{~h}, 24 \mathrm{~h}, 48 \mathrm{~h}$, $72 \mathrm{~h}$, or $96 \mathrm{~h}$. Then, $10 \mu \mathrm{l}$ of CCK- 8 reagent was added to each well and incubated for $3 \mathrm{~h}$ at $37{ }^{\circ} \mathrm{C}$. Finally, the absorbance was measured with an enzyme-labeled instrument (Thermo) at 450/650 $\mathrm{nm}$ excitation/emission wavelengths. The experiments were repeated at least in triplicate.

For colony formation, K-1 and BCPAP cells were seeded into 6-well cell plates (Corning Inc., Corning, USA) with a density of 500 cells/well in $2 \mathrm{ml}$ culture medium for 14 days. The culture medium was replaced once every 3-4 days. Colonies were washed with phosphate-buffered saline (PBS) 3 times, fixed with 4\% paraformaldehyde for 20 mins and stained by $0.5 \%$ crystal violet for $15 \mathrm{~min}$ at room temperature.

\section{Wound healing assay and transwell migration assay}

To measure the effect of IDH2 on cell migration, a wound healing assay was performed. Briefly, cells were seeded into 6-well plates and incubated until they were $100 \%$ confluent. The confluent monolayer of cells was scratched with a plastic apparatus to create a cell-free clear zone, about $1 \mathrm{~mm}$ in width. Then, the cells were washed with PBS and replenished with fresh culture medium. Cells were incubated for $0 \mathrm{~h}, 24 \mathrm{~h}$ or $48 \mathrm{~h}$, and the wound distance was measured regularly.
For the transwell migration assay, $15,000 \mathrm{~K}-1$ cells or 20,000 BCPAP cells were added into the top chamber of the insert well ( $8 \mu \mathrm{m}$ pore size; Corning, USA) with $200 \mu \mathrm{l}$ serum-free medium for $10 \mathrm{~h}$ or $12 \mathrm{~h}$. After removal of the non-migrating cells in the top chamber, the remaining cells were fixed with $4 \%$ paraformaldehyde for $20 \mathrm{~min}$, stained with $0.5 \%$ crystal violet for $15 \mathrm{~min}$ and counted.

\section{Statistical analysis}

SPSS for Windows version 22.0 (IBM Corporation, Armonk, NY, USA) was used for statistical analyses of data. Relapse-free survival and overall survival was defined as the duration of time between the date of the first surgery and the date of first local relapses and death, respectively. Survival curves were determined by plotting of Kaplan-Meier survival curves (Log-rank). For counting data,the values were expressed as the mean \pm SEM of at least three separate experiments. Independent $t$-test and Fisher's Exact test were used for calculating the significance between different groups. Statistical significance is indicated by $P<0.05$.

\section{Results \\ IDH2 expression and mutation in frozen tissues}

We examined the expression of key metabolic-related enzymes in thyroid cancer and adjacent normal tissues by qRT-PCR. We found asignificant mRNA expression increase on the level of IDH2 and PKM2 in thyroid cancer tissues than that in adjacent ones among these key metabolic-related enzymes (Figure S1A). In consideration of having been reported about the enzyme PKM2 in multiple cancers such as thyroid cancer,hepatocellular carcinoma and ovarian cancer,we selected the enzyme IDH2 for the following research. The expression levels of IDH2 in thyroid cancer and adjacent normal tissues was evaluated by qRT-PCR, immunohistochemical (IHC) staining and western blot analyses. In 19 of 21 paired samples of thyroid tissues, the mRNA level of IDH2 was found to be significantly higher in tumor than adjacent normal tissue by qRT-PCR, and the rest two paired samples with no significance (Figure 2A, Figure S1B, $P<0.01$ ). To verify the IDH2 levels, we performed western blot analysis with 8 pairs of matched thyroid cancer and adjacent normal tissues randomly selected from the 21 paired fresh tissues (Figure 2B). The results revealed that IDH2 was also significantly upregulated in 7 paired thyroid cancer 
tissues relative to adjacent normal ones at the protein level, and the rest one paired sample with no significance (Figure $2 \mathrm{C}, P<0.01)$. Then, we analyzed exon 4 of IDH2 gene for mutations in 21 frozen tissue samples from PTC patients. However, we found no mutations of IDH2 in PTC (data not shown).

\section{The clinical significance of IDH2 in thyroid cancer}

To investigate the expression pattern of IDH2 in thyroid cancer, we performed IHC staining in paraffin embedded tissues from thyroid cancer specimens. Tissue arrays were constructed from 187 thyroid cancer specimens of which 43 included matched adjacent normal tissues and were subjected to IHC staining. IDH2 protein was mainly distributed in the cytoplasm of cells (Figure 1). The thyroid cancer specimens were distinguished as 126 high expression and 61 low expression samples, while the adjacent normal tissues were distinguished as 6 high expression and 37 low expression according to the evaluation criteria. The high expression rate, or positive expression rate, was $14.0 \%$ and $67.4 \%$ in normal and tumor tissue, respectively, and the difference between these rates was significant (Figure 2D, $P<0.001$ ). Next, the correlations of IDH2 expression with the clinicopathological parameters of the patients as determined by the bilateral $\chi 2$ test are summarized in Table 1. Tumor size, $\mathrm{T}$ stage, Lymph node metastasis and TNM stage showed significant differences between the IDH2 high expression and low expression groups. However, age, gender, and multifocality did not show any significant differences between the two groups. Subsequently, we analyzed the correlation between clinicopathological features and the occurrence of neck lymph node metastasis, which is summarized in Table 1. Multiple

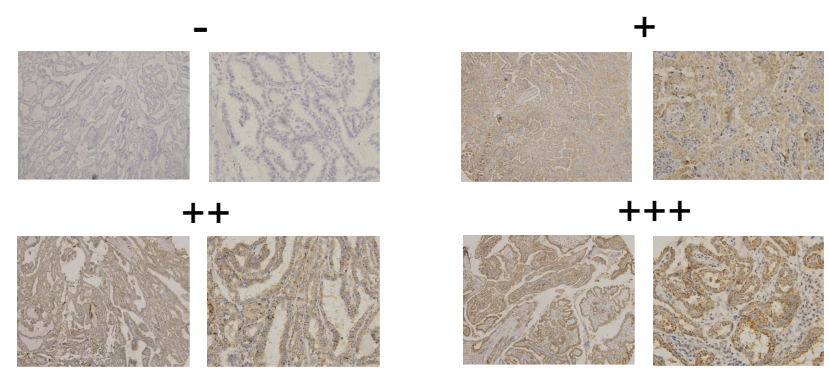

Figure I Representative immunohistochemical staining for IDH2 in thyroid tissues.

Notes: Tissue arrays were performed on 187 thyroid cancer specimens and 43 matched adjacent normal tissues by IHC staining. The final score was the multiplication product of these two scores. $0-1$ score $(-), 2-4$ scores $(+), 5-8$ scores $(++)$, and 9-12 scores $(+++)$. Overexpressed values are denoted by $(++)$ and $(+++)$. logistic regression analysis indicated that tumor size (OR 3.460; 95\% CI 1.761-6.797; $P<0.01)$ and IDH2 expression (OR 3.470; 95\% CI 1.711-7.040; $P<0.01$ ) were significantly correlated with the occurrence of neck LNM. However, the other five variants did not have significant differences, which means that these factors were not connected with LNM, which is summarized in Table 2.

\section{The effect of IDH2 expression in thyroid cells}

The effect of downregulation of IDH2 expression in thyroid cells

We detected the expression of IDH2 by immunoblotting in normal thyroid cell lines and thyroid cancer cell lines. We found that the expression level was higher in thyroid cancer cell lines than in normal thyroid cell lines, especially in BCPAP and $\mathrm{K}-1$ cell lines (Figure S1C). To investigate the potential function of IDH2 in thyroid cancer development and progression, we used lentiviralmediated sh-RNA (sh-IDH2-1/2) to construct BCPAP and $\mathrm{K}-1$ cell lines with stable IDH2 deletions. To assess transfection efficiency, we used qRT-PCR and western blot analysis to detect mRNA and protein expression levels of IDH2, respectively. The results indicated that the targeted shRNAs were able to effectively repress IDH2 at mRNA (Figure 3A, $P<0.01$ respectively) and protein levels (Figure 3B,C, $P<0.01$ and $P<0.05$ respectively) by shIDH2-1 and sh-IDH2-2 compared to sh-NC. To determine whether IDH2 promotes proliferation of human thyroid cells, we performed CCK8 and colony formation assays for proliferation to detect the effect of IDH2 on thyroid cancer cell lines. The results indicated that the OD450 values of the BCPAP and K-1 cells transfected with shIDH2-1 and sh-IDH2-2 were smaller compared with the sh-NC group (Figure 4A, $P<0.05$ respectively).

We then tested the migration capacity of IDH2transfectants using a wound healing assay, and the results showed that the migration ability of the sh-IDH2-1 and shIDH2-2 groups was significantly reduced relative to that of the sh-NC group. (Figure 4B). These results suggest that IDH2 can promote the migration of thyroid cancer cells. However, wound healing assays are primarily used to investigate the effects of cell-cell and cell-matrix interactions on cell migration, but do not reflect cell invasion capability. To better investigate this property we performed transwell invasion assays. The results indicated that the numbers of invasive BCPAP and K-1 cells transfected with sh-IDH2-1 and sh-IDH 2-2 were lower compared with the sh-NC group (Figure 4C). 

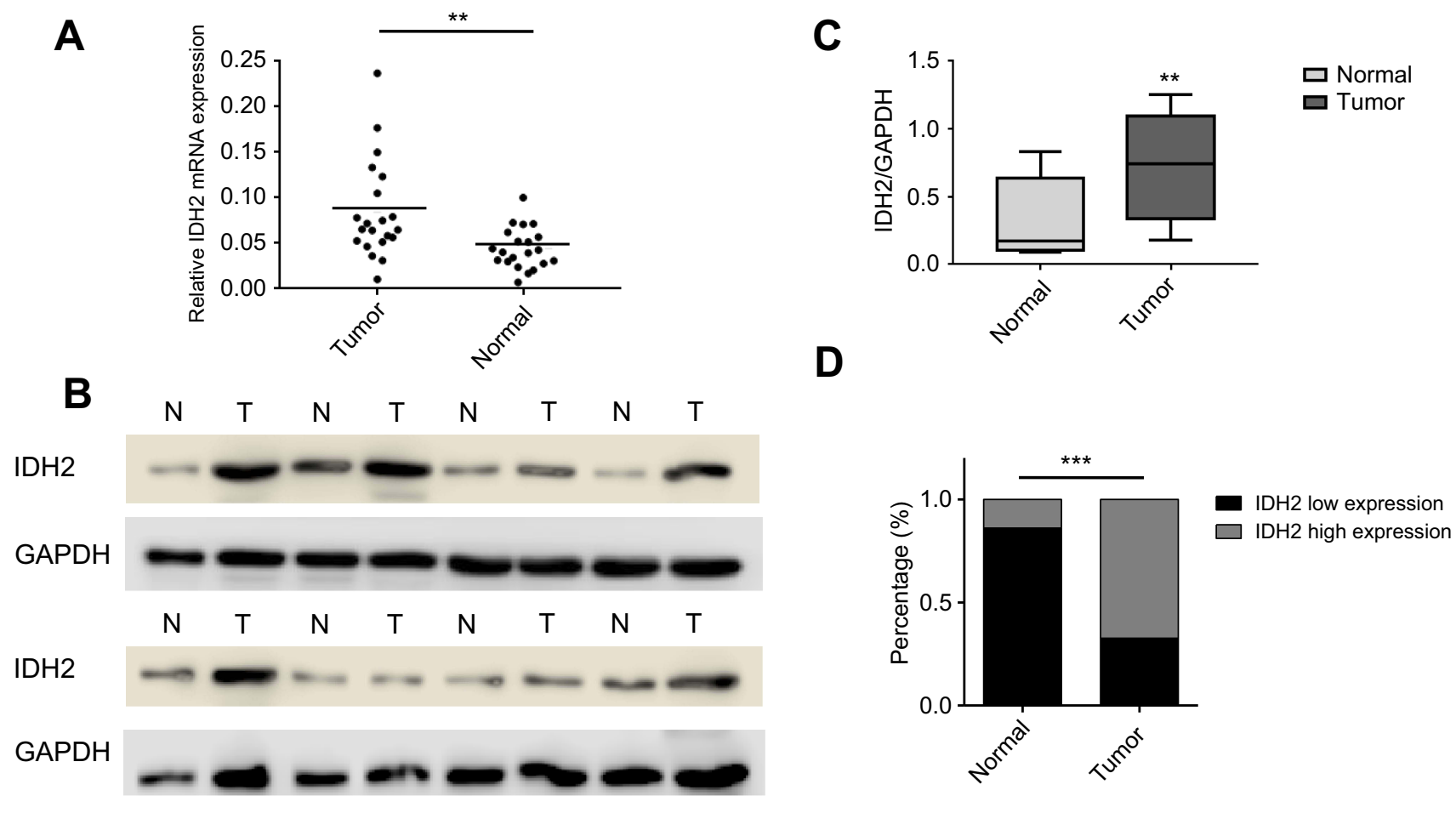

Figure 2 Expression levels of IDH2 in thyroid cancer and adjacent normal tissues.

Notes: (A) In 19 of 21 paired samples of thyroid tissues, it is shown that the mRNA level of IDH2 is significantly higher in tumor than adjacent normal tissue by quantitative real-time PCR. (B) Western blot analysis with eight pairs of thyroid cancer and adjacent normal tissues. (C) IDH2/GAPDH values of eight pairs of thyroid tissues. (D) Comparison of the expression of IDH2 immunohistochemistry in thyroid cancer and adjacent normal tissues. Values are expressed as mean \pm SD of three independent experiments. ${ }^{* *} p<0.0 \mathrm{I}, * * *<<0.00 \mathrm{I}$.

Abbreviations: $\mathrm{T}$, thyroid cancer tissues; $\mathrm{N}$, adjacent normal tissues.

Table I. Analysis of IDH2 expression and clinicopathological features in thyroid cancer

\begin{tabular}{|c|c|c|c|c|}
\hline \multirow[t]{2}{*}{ Clinicopathological features } & \multicolumn{2}{|l|}{ IDH2 expression } & \multirow[t]{2}{*}{$\chi^{2}$} & \multirow[t]{2}{*}{$P$-value } \\
\hline & High expression & Low expression & & \\
\hline \multicolumn{5}{|l|}{ Age } \\
\hline$<55$ & $103(81.7 \%)$ & $51(83.6 \%)$ & 0.098 & 0.754 \\
\hline$\geq 55$ & $23(\mid 8.3 \%)$ & $10(16.4 \%)$ & & \\
\hline \multicolumn{5}{|l|}{ Gender } \\
\hline Male & $38(30.2 \%)$ & $15(2436 \%)$ & 0.628 & 0.428 \\
\hline Female & $88(69.8 \%)$ & $46(75.4 \%)$ & & \\
\hline \multicolumn{5}{|l|}{ Tumor size $(\mathrm{cm})$} \\
\hline$>1$ & $88(69.8 \%)$ & $29(47.5 \%)$ & 8.727 & $0.003 * *$ \\
\hline$\leq 1$ & $38(30.2 \%)$ & $32(52.5 \%)$ & & \\
\hline \multicolumn{5}{|l|}{ Multifocality } \\
\hline Absent & $88(69.8 \%)$ & $40(65.6 \%)$ & 0.347 & 0.556 \\
\hline Present & $38(30.2 \%)$ & 21 (34.4\%) & & \\
\hline \multicolumn{5}{|l|}{$\mathbf{T}$ stage } \\
\hline TI-T2 & 110 (87.3\%) & $59(96.7 \%)$ & 4.192 & $0.04 I^{*}$ \\
\hline T3-T4 & $16(12.7 \%)$ & $2(3.3 \%)$ & & \\
\hline \multicolumn{5}{|l|}{ Lymph node metastasis } \\
\hline Absent & $48(38.1 \%)$ & $43(70.5 \%)$ & 17.267 & $<0.00 I^{* *}$ \\
\hline Present & 78 (6I.9\%) & 18 (29.5\%) & & \\
\hline \multicolumn{5}{|l|}{ TNM stage } \\
\hline I-II & II3 (89.7\%) & $61(100 \%)$ & 6.764 & $0.009 * *$ \\
\hline III-IV & $13(10.3 \%)$ & $0(0 \%)$ & & \\
\hline
\end{tabular}

Notes: Values are expressed as mean \pm SD of three independent experiments. $* P<0.05, * * P<0.01$. 
Table 2. Multivariate analysis of lymph node metastasis and clinicopathological features in thyroid cancer

\begin{tabular}{|l|l|l|l|l|l|l|l|}
\hline Characteristics & B & SE & Wald & P-value & OR & \multicolumn{2}{l|}{$95 \%$ CI } \\
\cline { 3 - 7 } & & & & & & Lower & Upper \\
\hline Gender & -0.293 & 0.364 & 0.649 & 0.420 & 0.746 & 0.365 & 1.522 \\
Age & -0.454 & 0.442 & 1.055 & 0.304 & 0.635 & 0.267 & 1.510 \\
Tumor size & 1.241 & 0.345 & 12.978 & $0.000 * * *$ & 3.460 & 1.761 & 6.797 \\
Multifocality & 0.264 & 0.352 & 0.564 & 0.453 & 1.302 & 0.654 & 2.595 \\
T stage & -0.113 & 0.555 & 0.41 & 0.839 & 0.893 & 0.301 & 2.651 \\
IDH2 expression & 1.244 & 0.361 & 11.887 & $0.001 * *$ & 3.470 & 1.711 & 7.040 \\
Constant & -0.773 & -1.134 & 0.464 & 0.496 & 0.462 & & \\
\hline
\end{tabular}

Notes: Values are expressed as mean \pm SD of three independent experiments. $* * P<0.01, * * * P<0.001$.

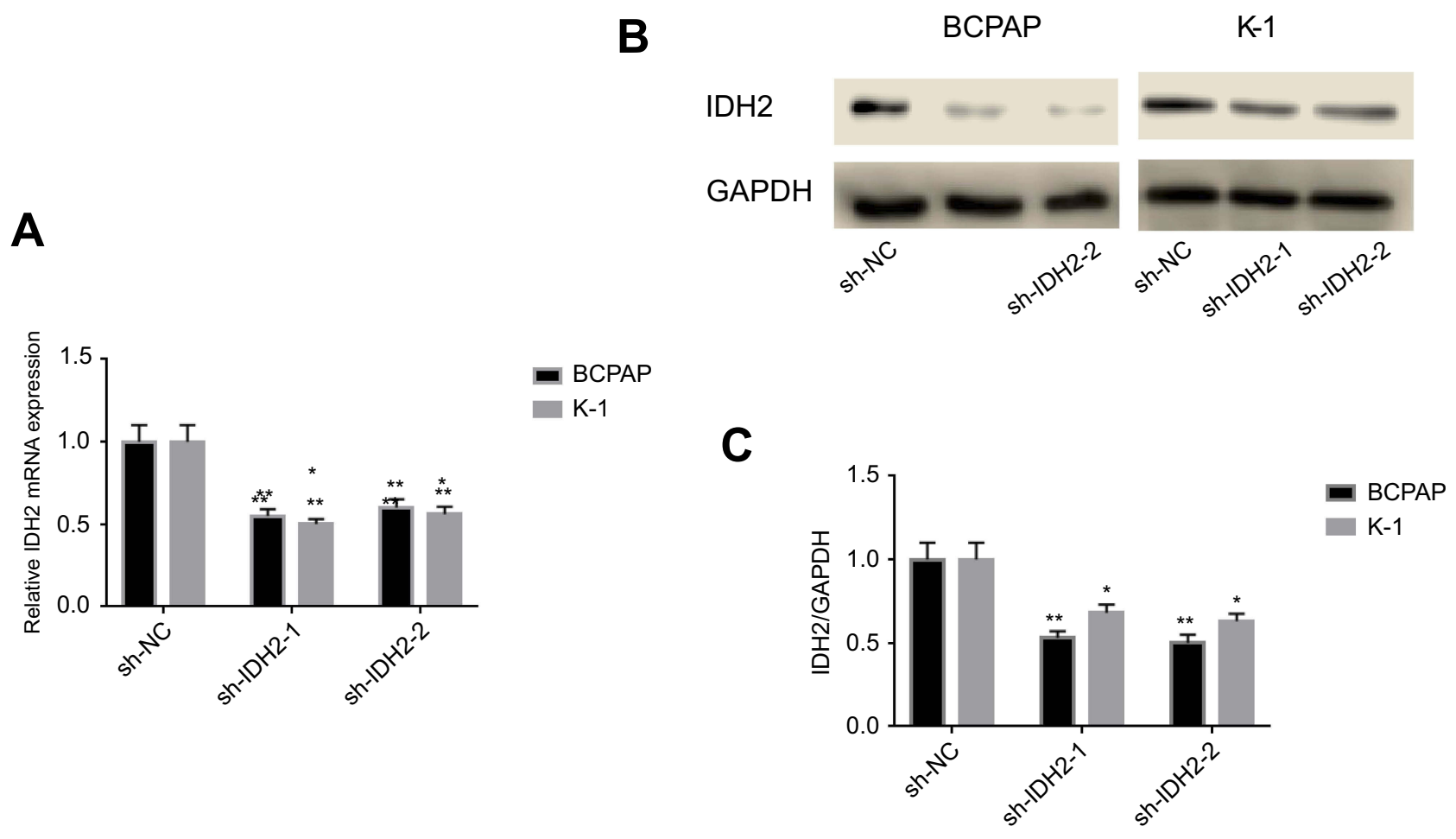

Figure 3 shRNA (sh-IDH2-I/2) to construct BCPAP and K-I cell lines with stable IDH2 deletion.

Notes: Quantitative real-time PCR (qRT-PCR) (A) and western blot (B) analysis to detect mRNA and protein expression levels of IDH2. (C) IDH2/GAPDH values of shIDH2-I/2. Values are expressed as mean qRT-PCR $(\mathbf{A})$ and western blot (B) analysis to detect mRNA and protein expression levels of IDH2. (C) IDH2/GAPDH values of sh$\mathrm{IDH} 2-\mathrm{I} / 2$. Values are expressed as mean \pm SD of three independent experiments. ${ }^{*} p<0.05,{ }^{* *} p<0.01 . \pm$ SD of three independent experiments. ${ }^{*} p<0.05$, ${ }^{* *} p<0.0 \mathrm{I}$.

Similarly, sh-IDH2-transfected thyroid cells showed less colony formation than sh-NC-transfected cells (Figure 4D). Taken together, these results indicate that IDH2 promotes proliferation, migration and invasiveness of thyroid cancer cells and may have a critical role in thyroid cancer progression.

Next, flow cytometry (FACS) was used to determine whether cell proliferation was reduced after IDH2 knockdown due to inhibition of cell cycle progression or cell death. The effects of IDH2 knockdown on the cellular features of apoptosis and cell cycle were examined by flow cytometry (FACS) experiments. The results indicated that apoptosis and cell cycle of IDH2 was not different between sh-IDH2 and sh-NC groups (Figure S2A,B).

\section{The effect of IDH2 overexpression in thyroid cells}

The targeted IDH2-plasmids were able to effectively upregulate IDH2 at the mRNA (Figure 5A, $P<0.001$ ) and protein level (Figure 5B,C, $P<0.01$ and $P<0.05$, respectively). The effect of IDH2 overexpression in thyroid cancer cells is shown in Figure 6. Compared to IDH2 vector-only control cells, both thyroid cancer cell lines 


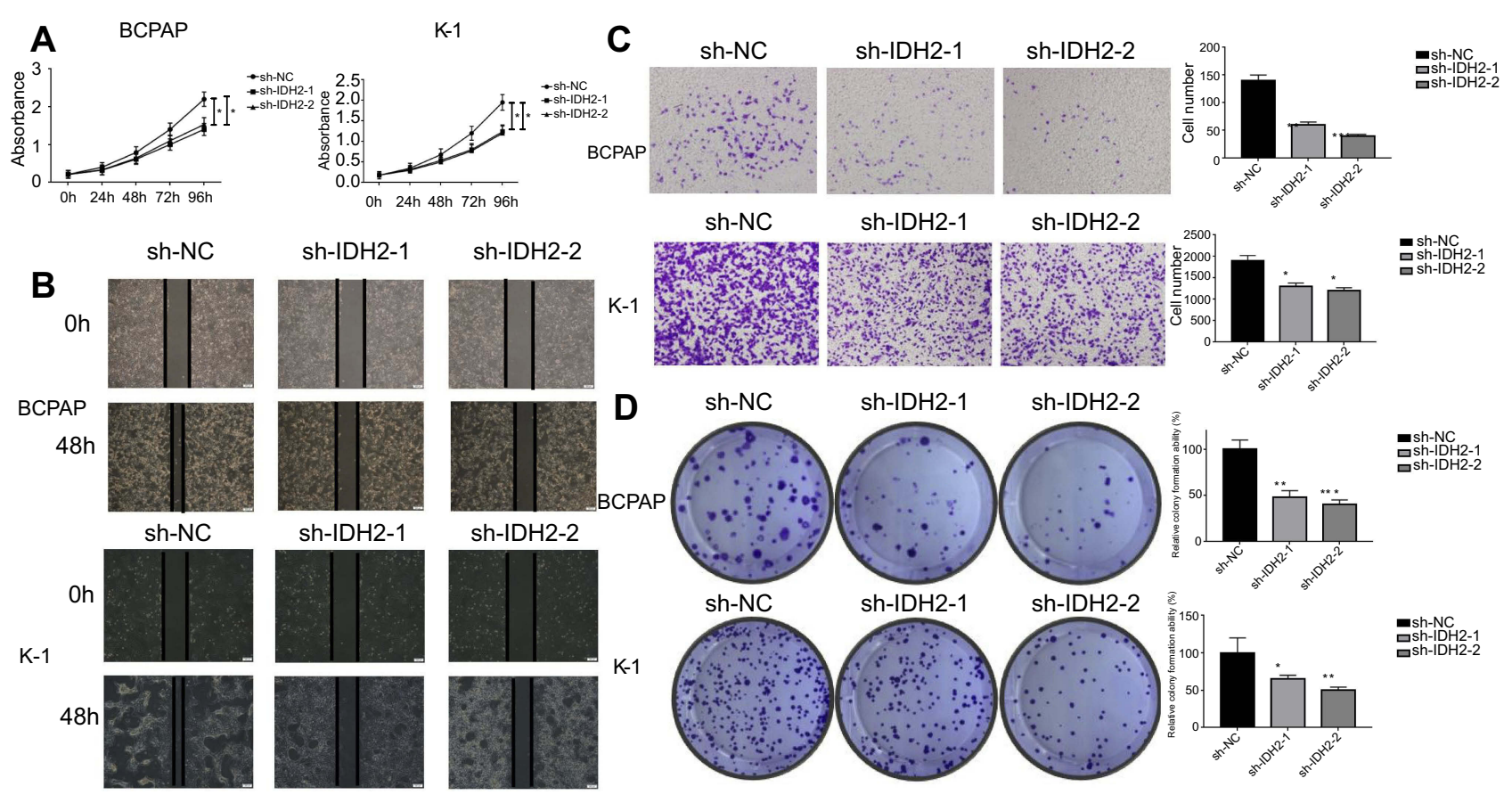

Figure 4 The effect of downregulation of IDH2 expression in BCPAP and K-I cell lines.

Notes: (A) The short-term effect of IDH2 expression on the tumor cell growth was evaluated by CCK-8. (B) Representative effect of IDH2 on tumor cell migration ability was evaluated by wound healing assay. (C) Representative results of transwell invasion assays and quantitative analysis. Values are expressed as mean \pm SD. of three independent experiments. $* p<0.05$. (D) Representative colony formation assay by monolayer culture and quantitative analysis. Values are expressed as mean \pm SD of three independent experiments. ${ }^{*} p<0.05, * * p<0.01$, $* * * p<0.001$.

B

\section{BCPAP}

IDH2

GAPDH

A
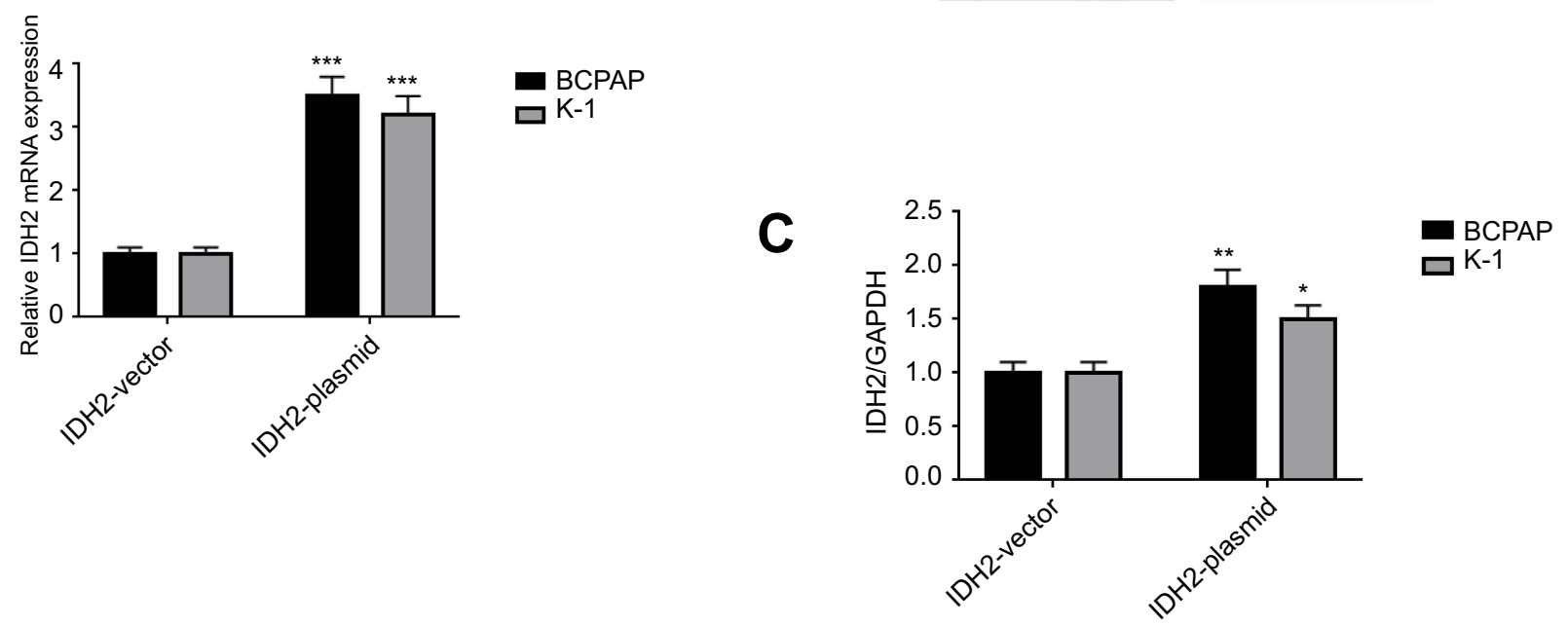

Figure 5 The targeted IDH2-plasmid was able to effectively upregulate IDH2 at mRNA and protein levels in BCPAP and K-I cell lines.

Notes: Quantitative real-time PCR (A) and western blot (B) analysis to detect mRNA and protein expression levels of IDH2. (C) IDH2/GAPDH values of IDH2 overexpression. Values are expressed as mean \pm SD of three independent experiments. ${ }^{*} p<0.05, * *_{p}<0.0$ I, $*_{* *} p<0.00$ I. 


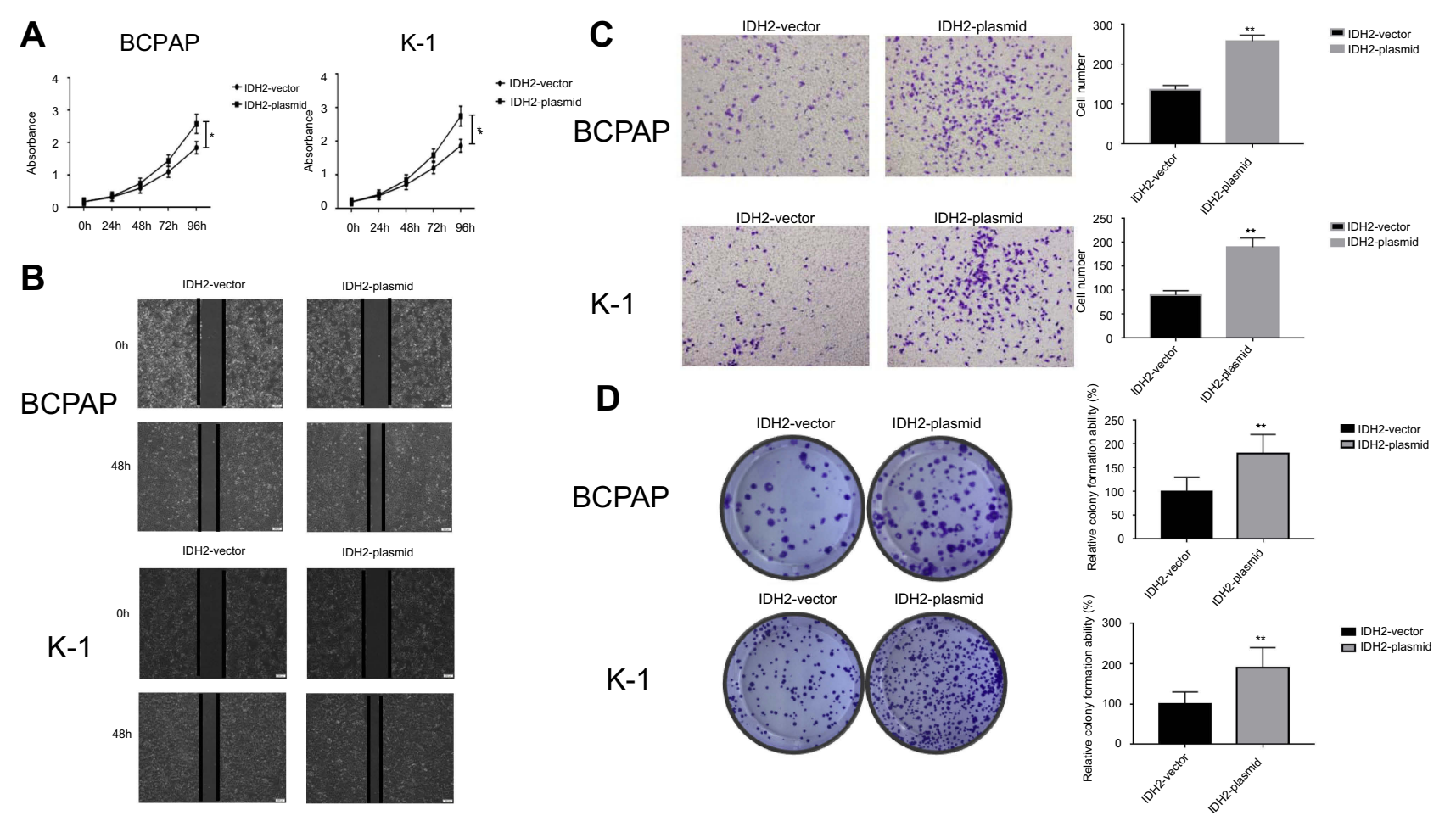

Figure 6 The effect of IDH2 overexpression in thyroid cells.

Notes: (A) The short-term effect of IDH2 expression on the tumor cell growth was evaluated by CCK-8. (B) Representative effect of IDH2 on tumor cell migration ability was evaluated by wound healing assay. (C) Representative results of transwell invasion assays and quantitative analysis. (D) Representative colony formation assay by monolayer culture and quantitative analysis. Values are expressed as mean \pm SD of three independent experiments. ${ }^{*} p<0.05$, $* * p<0.01$.

with IDH2 overexpression had faster growth rates as evaluated by CCK8 assay (Figure $6 \mathrm{~A}, P<0.05$ ). In addition, the result also indicated that IDH2-plasmid transfection increased the migration capacity and invasion capability of thyroid cancer cells compared with the IDH2-vector group by wound-healing and transwell invasion assays (Figure 6B,C, $P<0.01$, respectively). Colony formation assays further showed that IDH 2 overexpression increased the number of colonies formed (Figure 6D, $P<0.01$, respectively). Taken together, these experiments suggested that IDH2 promotes thyroid cancer cell proliferation and invasion.

\section{Discussion}

The mechanism of IDH1 or IDH2 mutations leading to human cancer remains to be fully elucidated. IDH2 plays an important role in antioxidant deficiency, leading to lipid peroxidation, oxidative DNA damage, and intracellular peroxide production. The state of IDH2 mutants in cancer has been revealed in recent years. However, IDH2 mutations are not considered to be common mutation sites in patients with thyroid cancer according to previous reports. $^{25,31}$ Further, in our study, the IDH2 gene was examined for mutations in thyroid cancer, but no mutations were found. Thyroid cancer is one of the most prevalent human cancers. The role of wild-type IDH2 in carcinoma remains controversial, and there are different biological characteristics in different carcinomas. ${ }^{27,28,32-35}$ However, the significance and expression of IDH2 in thyroid cancer is still unknown. Consequently, current research only focuses on the role of wild-type IDH2 in thyroid cancer.

In the present study, we report for the first time the expression levels of IDH2 in thyroid cancer and adjacent normal tissues by qRT-PCR, IHC staining and western blot analyses. It was demonstrated that the mRNA and protein levels of IDH2 were significantly higher in tumor than adjacent normal tissue. This indicates that IDH2 may play a very important role in the development and progression of thyroid cancer. In addition, the relationship between IDH2 and clinicopathologic factors and prognosis varies between tumors. ${ }^{27,32,34,35}$ In our study, tumor size, T stage, lymph node metastasis and TNM stage showed significant differences between IDH2 high expression and low expression groups. However, age, gender, and multifocality did not show any significant differences between 
the two groups. Multiple logistic regression analysis indicated that tumor size (OR 3.460; 95\% CI 1.761-6.797; $P<0.01$ ) and IDH2 expression (OR 3.470; 95\% CI 1.711-7.040; $P<0.01)$ were significantly connected with the occurrence of neck LNM. These results demonstrate the potential role of IDH2 in the biological mechanisms of thyroid carcinogenesis and progression and also indicate IDH2 as a thyroid cancer oncogene.

The mechanisms of IDH2 regulation and effects on thyroid cancer cell have not been elucidated. According to analysis of the correlations of IDH2 expression with the clinicopathological parameters of the patients, IDH2 may play an important role in the development of thyroid cancer. To test this hypothesis and verify the cancerpromoting effect of IDH2 in vitro, we used lentiviralmediated shRNA (sh-IDH2-1/2) or transient transfection of IDH2 plasmids to construct BCPAP and K-1 cell lines with stable IDH2 deletion or overexpression, respectively. We then used these cells to investigate the effect of IDH2 on cell proliferation in CCK8 and colony forming experiments and the migration capacity and invasion capability by wound-healing assay and transwell invasion assays, respectively. We observed that shRNA inhibits cell proliferation, migration, and invasion and these capabilities are promoted by transfection of the IDH2 overexpression plasmid. These results suggest that the expression level of IDH2 may play a crucial role in the proliferation, migration and invasion of thyroid cancer cells.

IDH 2 converts isocitrate to $\alpha-\mathrm{KG}$ through the reduction of NADP + to NADPH, and mutations of IDH1 and IDH2 exhibited neomorphic enzymatic activity, resulting in the production of an onco-metabolite named 2-hydroxyglutarate $(2-\mathrm{HG}) .{ }^{19}$ It has been shown that $2-\mathrm{HG}$ can impair histone demethylation and switch cell differentiation pathways. $^{20}$ In addition, a reversible reduction of a-KG to isocitrate has also been discovered under hypoxic conditions with wild-type IDH1 and IDH2, and an elevation of 2-HG levels was also detected in this process. ${ }^{36}$ The regulatory mechanism of IDH2 and its effect on cancer have not been clarified. Mitochondrial NADP+-dependent isocitrate dehydrogenase plays an important role in cellular defense against oxidative damage by providing the NADPH needed to produce glutathione. ${ }^{37,38} \mathrm{GSH}$ and Trx are important antioxidant systems that protect cells from oxidative damage and xenobiotic toxicity by removing reactive oxygen species (ROS). ${ }^{39}$ Cellular oxidative stress arising from high levels of ROS contributes to the development and progression of malignant tumors, including carcinogenesis, aberrant growth, angiogenesis and metastasis. ${ }^{40}$ Furthermore, GSH and Trx systems also promote cancer cell growth and suppress immune responses. ${ }^{41}$ IDH2 plays an important role in antioxidant deficiency and leads to increased lipid peroxidation, oxidative DNA damage; intracellular peroxide generation. ${ }^{37,42}$ Therefore, IDH2 may affect cell proliferation through alterations in NADP and 2-HG levels.

The limitation of this study is that it is a retrospective study which includes a relatively small number of patient specimens. Further research is needed to confirm the precise molecular regulation of IDH2 in cell growth and its effects on cell invasion. Despite these limitations, our results indicate that IDH2 may play a very important role in the development and progression of thyroid cancer and also indicates IDH2 as a thyroid cancer oncogene. IDH2 can also be used as a potential biomarker for diagnosis and prognosis and may be a potential therapeutic target for thyroid cancer. Studying the role of IDH2 in thyroid cancer may still be valuable.

\section{Conclusion}

IDH2 promotes the proliferation, migration and invasion of papillary thyroid carcinoma, may play an important role in the development of thyroid cancer. And IDH2 can be used as a potential biomarker for diagnosis and prognosis and may be a potential therapeutic target for thyroid cancer.

\section{Acknowledgment}

This work was supported by research grant from National Scientific Foundation of China (Grant No. 81702629).

\section{Disclosure}

The authors have no conflicts of interest in this work.

\section{References}

1. Davies L, Welch HG. Current thyroid cancer trends in the United States. JAMA Otolaryngol Head Neck Surg. 2014;140(4):317-322. doi:10.1001/jamaoto.2014.1

2. Pellegriti G, Frasca F, Regalbuto C, Squatrito S, Vigneri R. Worldwide increasing incidence of thyroid cancer: update on epidemiology and risk factors. J Cancer Epidemiol. 2013;2013:965212. doi:10.1155/ 2013/965212

3. Yu GP, Li JC, Branovan D, McCormick S, Schantz SP. Thyroid cancer incidence and survival in the national cancer institute surveillance, epidemiology, and end results race/ethnicity groups. Thyroid. 2010;20(5):465-473. doi:10.1089/thy.2008.0281 
4. Rahib L, Smith BD, Aizenberg R, Rosenzweig AB, Fleshman JM, Matrisian LM. Projecting cancer incidence and deaths to 2030: the unexpected burden of thyroid, liver, and pancreas cancers in the United States. Cancer Res. 2014;74(11):2913-2921. doi:10.1158/ 0008-5472.CAN-14-0155

5. ACS. American Cancer Society: cancer facts and figures. 2016;2016.

6. Hundahl SA, Fleming ID, Fremgen AM, Menck HR. A national cancer data base report on 53,856 cases of thyroid carcinoma treated in the U.S., 1985-1995 [see commetns]. Cancer. 1998;83(12):2638-2648.

7. Oh CM, Won YJ, Jung KW, et al. Cancer statistics in Korea: incidence, mortality, survival, and prevalence in 2013. Cancer Res Treat. 2016;48(2):436-450. doi:10.4143/crt.2016.089

8. Cooper DS, Doherty GM, Haugen BR, et al. Management guidelines for patients with thyroid nodules and differentiated thyroid cancer Thyroid. 2006;16(2):109-142. doi:10.1089/thy.2006.16.109

9. Grant CS. Recurrence of papillary thyroid cancer after optimized surgery. Gland Surg. 2015;4(1):52-62. doi:10.3978/j.issn.2227-684X.2014.12.06

10. Carneiro RM, Carneiro BA, Agulnik M, Kopp PA, Giles FJ. Targeted therapies in advanced differentiated thyroid cancer. Cancer Treat Rev. 2015;41(8):690-698. doi:10.1016/j.ctrv.2015.06.002

11. Kojic KL, Kojic SL, Wiseman SM. Differentiated thyroid cancers: a comprehensive review of novel targeted therapies. Expert Rev Anticancer Ther. 2012;12(3):345-357. doi:10.1586/era.12.8

12. Schlumberger M, Sherman SI. Clinical trials for progressive differentiated thyroid cancer: patient selection, study design, and recent advances. Thyroid. 2009;19(12):1393-1400. doi:10.1089/thy.2009.1603

13. Jose C, Bellance N, Rossignol R. Choosing between glycolysis and oxidative phosphorylation: a tumor's dilemma? Biochimica et biophysica acta. Jun. 2011;1807(6):552-561.

14. Koppenol WH, Bounds PL, Dang CV. Otto Warburg's contributions to current concepts of cancer metabolism. Nature Rev Cancer. 2011;11(5):325-337. doi:10.1038/nrc3038

15. Dang L, Yen K, Attar EC. IDH mutations in cancer and progress toward development of targeted therapeutics. Annals oncol. 2016;27 (4):599-608. doi:10.1093/annonc/mdw013

16. Zhao S, Lin $\mathrm{Y}, \mathrm{Xu} \mathrm{W}$, et al. Glioma-derived mutations in IDH1 dominantly inhibit IDH1 catalytic activity and induce HIF-1alpha. Science. 2009;324(5924):261-265. doi:10.1126/science.1170944

17. Gross S, Cairns RA, Minden MD, et al. Cancer-associated metabolite 2-hydroxyglutarate accumulates in acute myelogenous leukemia with isocitrate dehydrogenase 1 and 2 mutations. J Exp Med. 2010;207 (2):339-344. doi:10.1084/jem.20092506

18. Dang L, White DW, Gross S, et al. Cancer-associated IDH1 mutations produce 2-hydroxyglutarate. Nature. 2010;465(7300):966. doi:10.1038/nature09132

19. Ward PS, Patel J, Wise DR, et al. The common feature of leukemia-associated IDH1 and IDH2 mutations is a neomorphic enzyme activity converting alpha-ketoglutarate to 2-hydroxyglutarate. Cancer Cell. 2010;17(3):225-234. doi:10.1016/j.ccr.2010.01.020

20. Lu C, Ward PS, Kapoor GS, et al. IDH mutation impairs histone demethylation and results in a block to cell differentiation. Nature. 2012;483(7390):474-478. doi:10.1038/nature10860

21. Xu W, Yang H, Liu Y, et al. Oncometabolite 2-hydroxyglutarate is a competitive inhibitor of alpha-ketoglutarate-dependent dioxygenases. Cancer Cell. 2011;19(1):17-30. doi:10.1016/j. ccr.2010.12.014

22. Yan H, Parsons DW, Jin G, et al. IDH1 and IDH2 mutations in gliomas. $N$ Engl $J$ Med. 2009;360(8):765-773. doi:10.1056/ NEJMoa0808710

23. Marcucci G, Maharry K, Wu YZ, et al. IDH1 and IDH2 gene mutations identify novel molecular subsets within de novo cytogenetically normal acute myeloid leukemia: a Cancer and Leukemia Group B study. J clin oncol. 2010;28(14):2348-2355. doi:10.1200/JCO.2009.27.3730

24. Fujii T, Khawaja MR, DiNardo CD, Atkins JT, Janku F. Targeting isocitrate dehydrogenase (IDH) in cancer. Discov Med. 2016;21 (117):373-380.
25. Murugan AK, Bojdani E, Xing M. Identification and functional characterization of isocitrate dehydrogenase 1 (IDH1) mutations in thyroid cancer. Biochem Biophys Res Commun. 2010;393 (3):555-559. doi:10.1016/j.bbrc.2010.02.095

26. Altenberg B, Greulich KO. Genes of glycolysis are ubiquitously overexpressed in 24 cancer classes. Genomics. 2004;84 (6):1014-1020. doi:10.1016/j.ygeno.2004.08.010

27. Guirguis A, Elishaev E, Oh SH, Tseng GC, Zorn K, DeLoia JA. Use of gene expression profiles to stage concurrent endometrioid tumors of the endometrium and ovary. Gynecol Oncol. 2008;108 (2):370-376. doi:10.1016/j.ygyno.2007.10.008

28. Wang WZ, Guo X, Duan C, et al. Comparative analysis of gene expression profiles between the normal human cartilage and the one with endemic osteoarthritis. Osteoarthritis Cartilage. 2009;17 (1):83-90. doi:10.1016/j.joca.2008.05.008

29. Tian GY, Zang SF, Wang L, Luo Y, Shi JP, Lou GQ. Isocitrate dehydrogenase 2 suppresses the invasion of hepatocellular carcinoma cells via matrix metalloproteinase 9. Cell Physiol Biochem. 2015;37 (6):2405-2414. doi:10.1159/000438593

30. Wu D. Isocitrate dehydrogenase 2 inhibits gastric cancer cell invasion via matrix metalloproteinase 7. Tumour Biol. 2016;37(4):5225-5230. doi:10.1007/s13277-015-4358-2

31. Bleeker FE, Lamba S, Leenstra S, et al. IDH1 mutations at residue p. R132 (IDH1(R132)) occur frequently in high-grade gliomas but not in other solid tumors. Hum Mutat. 2009;30(1):7-11. doi:10.1002/ humu.20937

32. Chen $\mathrm{X}, \mathrm{Xu} \mathrm{W}$, Wang $\mathrm{C}$, et al. The clinical significance of isocitrate dehydrogenase 2 in esophageal squamous cell carcinoma. Am $J$ Cancer Res. 2017;7(3):700-714.

33. Liu WR, Tian MX, Jin L, et al. High expression of 5-hydroxymethylcytosine and isocitrate dehydrogenase 2 is associated with favorable prognosis after curative resection of hepatocellular carcinoma. J Exp Clin Cancer Res. 2014;33:32. doi:10.1186/ 1756-9966-33-32

34. Lv Q, Xing S, Li Z, et al. Altered expression levels of IDH2 are involved in the development of colon cancer. Exp Ther Med. 2012;4 (5):801-806. doi:10.3892/etm.2012.676

35. Memon AA, Chang JW, Oh BR, Yoo YJ. Identification of differentially expressed proteins during human urinary bladder cancer progression. Cancer Detect Prev. 2005;29(3):249-255. doi:10.1016/ j.cdp.2005.01.002

36. Wise DR, Ward PS, Shay JE, et al. Hypoxia promotes isocitrate dehydrogenase-dependent carboxylation of alpha-ketoglutarate to citrate to support cell growth and viability. Proc Natl Acad Sci USA. 2011;108(49):19611-19616. doi:10.1073/pnas.1117773108

37. Nakamura H. Thioredoxin and its related molecules: update 2005. Antioxid Redox Signal. 2005;7(5-6):823-828. doi:10.1089/ ars.2005.7.823

38. Avery AM, Willetts SA, Avery SV. Genetic dissection of the phospholipid hydroperoxidase activity of yeast gpx3 reveals its functional importance. J Biol Chem. 2004;279(45):46652-46658. doi:10.1074/ jbc.M408340200

39. Lu J, Holmgren A. Thioredoxin system in cell death progression. Antioxid Redox Signal. 2012;17(12):1738-1747. doi:10.1089/ ars.2012.4650

40. Kim S, Kim SY, Ku HJ, et al. Suppression of tumorigenesis in mitochondrial $\mathrm{NADP}(+)$-dependent isocitrate dehydrogenase knock-out mice. Biochim Biophys Acta. 2014;1842(2):135-143. doi:10.1016/j.bbadis.2013.11.008

41. Benhar M, Shytaj IL, Stamler JS, Savarino A. Dual targeting of the thioredoxin and glutathione systems in cancer and HIV. J Clin Invest. 2016;126(5):1630-1639. doi:10.1172/ JCI85339

42. Geisbrecht BV, Gould SJ. The human PICD gene encodes a cytoplasmic and peroxisomal NADP(+)-dependent isocitrate dehydrogenase. J Biol Chem. 1999;274(43):30527-30533. 


\section{Supplementary material}

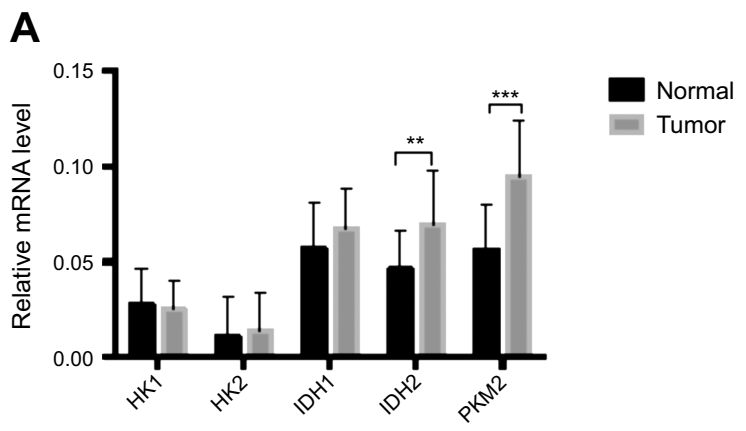

B

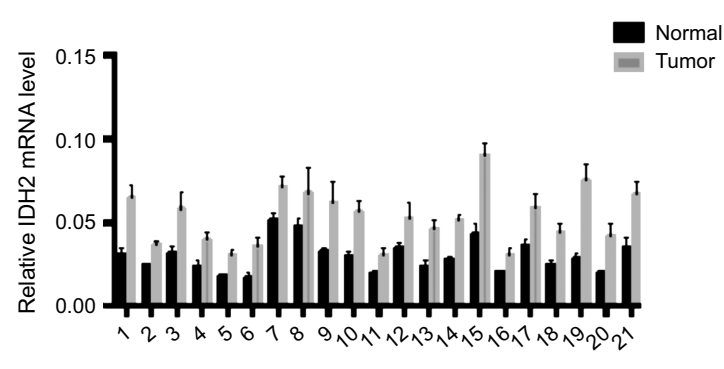

C

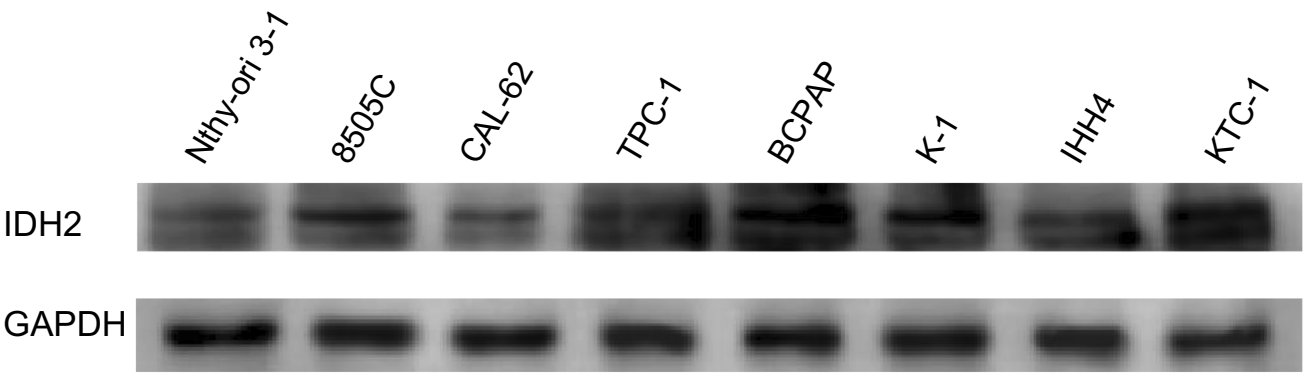

Figure SI The expression of key metabolic-related enzymes and IDH2 in thyroid cancer and adjacent normal tissues and cell lines.

Notes: (A) The expression of key metabolic-related enzymes in thyroid cancer and adjacent normal tissues by quantitative real-time PCR (qRT-PCR). (B) The mRNA level of IDH2 in 21 paired samples of thyroid tissues by qRT-PCR. (C) The expression of IDH2 by immunoblotting in normal thyroid cell lines and thyroid cancer cell lines. Values are expressed as mean $\pm S D$ of three independent experiments. $* * P<0.01$, *** $P<0.001$.

A
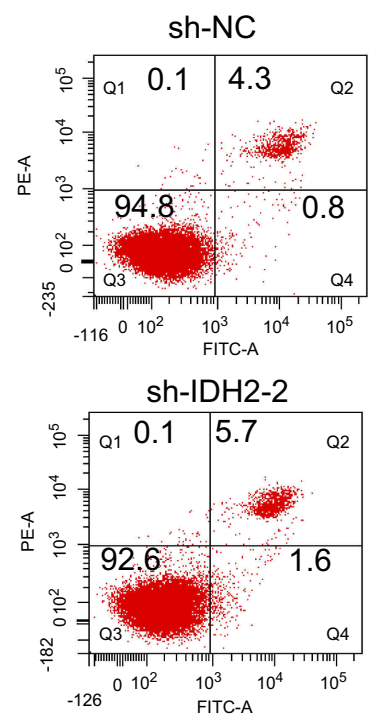

B
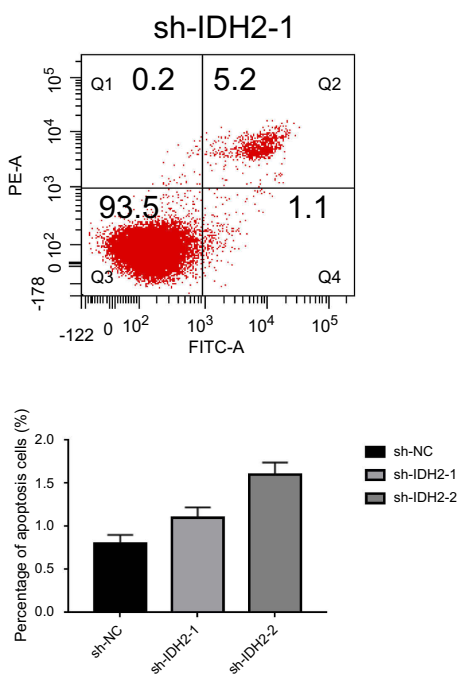

sh-NC

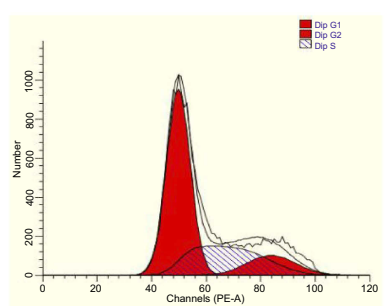

sh-IDH2-2

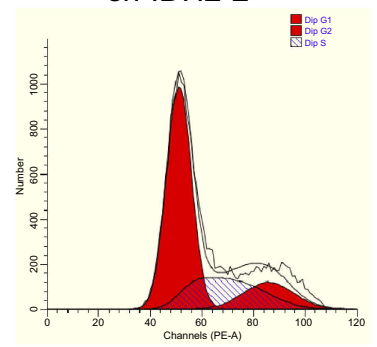

sh-IDH2-1
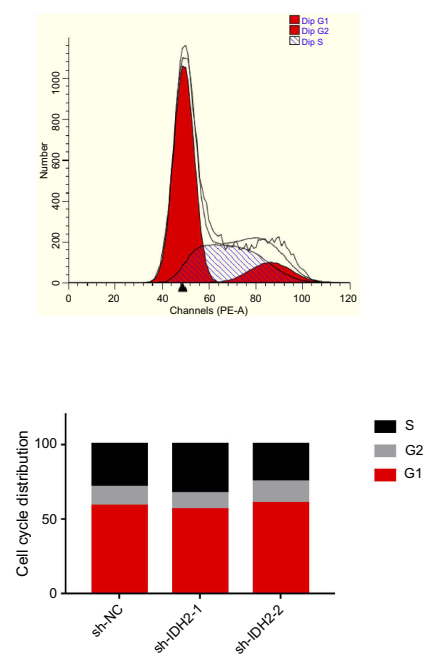

Figure S2 The effects of IDH2 knockdown on the cellular features of apoptosis (A) and cell cycle (B) were examined by flow cytometry experiments. 


\section{Publish your work in this journal}

Cancer Management and Research is an international, peer-reviewed open access journal focusing on cancer research and the optimal use of preventative and integrated treatment interventions to achieve improved outcomes, enhanced survival and quality of life for the cancer patient.

The manuscript management system is completely online and includes a very quick and fair peer-review system, which is all easy to use. Visit http://www.dovepress.com/testimonials.php to read real quotes from published authors. 